\title{
Effects of polyglecaprone 25, silk and catgut suture materials on oral mu- cosa wound healing in diabetic rats: An evaluation of nitric oxide dynamics
}

\author{
Nergiz Yilmaz ${ }^{1}$, Samet İnal ${ }^{2}$, Mehtap Muğlali ${ }^{3}$, Tolga Güvenç ${ }^{4}$, Burcu Baş ${ }^{5}$
}

\footnotetext{
${ }^{1}$ Assoc. Prof. Dr. Ondokuz Mayıs University, Faculty of Dentistry, Department of Oral and Maxillofacial Surgery, Samsun, Turkey

${ }^{2}$ Dr. Ondokuz Mayıs University, Faculty of Dentistry, Department of Oral and Maxillofacial Surgery, Samsun, Turkey

${ }^{3}$ Assoc. Prof. Dr. Ondokuz Mayıs University, Faculty of Dentistry, Department of Oral and Maxillofacial Surgery, Samsun, Turkey

${ }^{4}$ Prof. Dr. Ondokuz Mayıs University, Faculty of Veterinarian, Department of Pathology, Samsun, Turkey

${ }^{5}$ Asisstant Prof. Ondokuz Mayıs University, Faculty of Dentistry, Department of Oral and Maxillofacial Surgery, Samsun, Turkey
}

Correspondence:

Ondokuz Mayls Üniversitesi, Diş Hekimligi Fakültesi

Ağız Diş Çene Hastalıkları ve Cerrahisi AD

55139 Kurupelit / Samsun / Turkey

nergizy@omu.edu.tr

Received: $12 / 05 / 2009$

Accepted: 20/09/2009
Yilmaz N, İnal S, Muğlali M, Güvenç T, Baş B. Effects of polyglecaprone 25 , silk and catgut suture materials on oral mucosa wound healing in diabetic rats: An evaluation of nitric oxide dynamics. Med Oral Patol Oral Cir Bucal. 2010 May 1;15 (3):e526-30.

http://www.medicinaoral.com/medoralfree01/v15i3/medoralv15i3p526.pdf

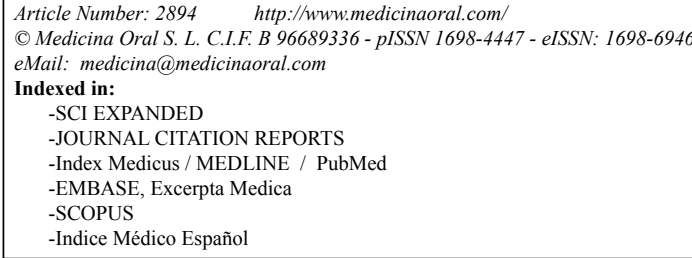

\begin{abstract}
Objectives: The purpose of this study is to investigate the effects of monocryl (Polyglecaprone 25), silk and catgut suture materials on wound healing in diabetic rats and to evaluate NO (nitric oxide) dynamics.

Study desing: Fourty-eight male Wistar-Albino rats weighting 220-270 g were used in this study. The rats were categorized into 2 groups, as control group $(n=24)$ and streptozotocin (STZ) induced diabetic group $(n=24)$. For each group, incision wounds were created on the inner cheeck mucosa of the animals and wounds were closed primarily with three different types of sutures. These materials were sized 3-0 monocryl (polyglecaprone 25), silk and catgut sutures swaged on. Excisional biopsies were performed at 2nd, 7th, 14th and 21st day (2 rats per day) from the initial surgical procedure. Effects of these suture materials on wound healing and NOS (nitric oxide synthase) activities were compared immunohistochemically in the study. eNOS and iNOS (Endothelial and induced nitric oxide synthase) activities were classified as mild $(+)$, moderate $(++)$ and severe $(+++)$.

Results: Mild eNOS activities were seen in both diabetic and control groups at 2 nd day specimens. Activities of silk and catgut were similar both in diabetic and control groups at 14th day, this activity in monocryl was mild. 21st day specimens revealed that monocryl has mild eNOS activity than silk and catgut sutures. iNOS activities in diabetic group were greater than control groups and the degrees of the activity in monocryl, silk and catgut sutures were mild, severe and moderate at the 7th day. At the 14th day there was no iNOS activity in diabetic monocryl group.

Conclusion: All of the materials have the potential capacity to be well tolerated however monocryl seemed to have more benefical effects on wound healing in diabetic subjects.
\end{abstract}

Key words: Wound healing, diabetes, nitric oxide, suture. 


\section{Introduction}

Wound repair is a well orchestrated and highly coordinated process that includes a series of overlapping phases: inflammation, cell proliferation, matrix deposition, and tissue remodeling (1). Sutures play an important role in wound healing after surgical interventions and thus the selection of suture material, especially in oral procedures, must be made carefully. This location differs from other body sites due to the constant presence of saliva, spesific microbiata, high vascularization, as well as its functions related to speech, mastication and swallowing (2).

The series of pathological changes associated with several diseases ultimately leads to severely disturbed wound healing conditions (3). Systemic diseases which delay wound healing is another significant point that effects the choice of suture material and represent major clinical importance (2). Diabetic wound healing impairment is one of the most well-known chronic wound situations (4). The factors ensuring appropriate intercellular communication during wound repair are not completely understood. Although protein-type mediators are wellestablished players in this process, emerging evidence from both animal and human studies indicates that NO (nitric oxide) plays a key role in wound repair (5).

Nitric oxide is an intracellular messenger molecule with important immune functions (6). It is produced by a group of isoenzymes collectively termed NOS (NO synthase) (7). Three distinct isoforms of NOS have been explained: the endothelial (eNOS), neuronal (nNOS), and inducible (iNOS) NOS. Immunohistochemical investigations have been performed using iNOS and eNOS as immunohistochemical markers, since many studies have shown the importance of these enzymes that have a role in a nonspecific immune response, acting as a toxic agent in infections (6).

The aim of this study is to investigate the effects of monocryl (Polyglecaprone 25), silk and catgut suture materials on wound healing in diabetic rats and to evaluate NO dynamics.

\section{Material and Methods}

Animals: Fourty-eight male Wistar-Albino rats weighting 220-270 $\mathrm{g}$ were used in this study. The animals were kept in a room which had a constant temperature of $22 \pm 1^{\circ} \mathrm{C}$ with a 12-hour-light and 12-hour-darkness cycle. The rats were housed 2 animals per cage in a room and fed specific rat chow and water ad libitum. All experiments were performed at the surgical research laboratory of Ondokuz Mayıs University Faculty of Medicine. All procedures in this experiment were conducted according to the guidelines approved by the Animal Etchical Committee of Ondokuz Mayıs University. The rats were categorized into 2 groups, as control group $(\mathrm{n}=24)$ and streptozotocin (STZ) induced diabetic group $(\mathrm{n}=24)$.
Model of induced diabetes: Diabetes was induced by intraperitoneal injection of streptozotocin (STZ) (Sigma Chemical Co., St. Louis, MO, USA) (40 mg/kg in $0.2 \mathrm{ml}$ of $10 \mathrm{mM}$ citrate buffer, $\mathrm{pH} 4.5$ ) After two weeks, blood glucose levels were assessed and all the rats those blood glucose level greater than $250 \mathrm{mg} / \mathrm{dl}$ were defined as diabetic in the study (measured by glucometer).

Surgical Procedure: Anesthesia was performed by intraperitoneal injection Ksilazin hydrochlorid 0.1-0.2 $\mathrm{mg} / \mathrm{kg}$ IM for $45 \mathrm{~min}$., ketamine $10-15 \mathrm{mg} / \mathrm{kg}$ IM. For each group, incision wounds of equal length those were $1,5 \mathrm{~cm}$, were created on the inner cheeck mucosa of the animals and wounds were closed primarily with three different types of sutures. These materials were size 3-0 monocryl, silk and catgut sutures swaged on. Eight rats were used in each group and excisional biopsies were performed at $2 \mathrm{nd}, 7$ th, 14 th and 21 st day (2 rats per day) from the initial surgical procedure.

Histotopathological Examination: Tissue specimens were fixed in $10 \%$ formalin, dehydrated, cleared, and embedded in paraffin. The paraffin-embedded specimens were cut with a microtome at $5 \mu \mathrm{m}$ thickness. The sections were stained with hematoxylin and eosin (HE) for histopathological evaluation.

eNOS and iNOS immunohistochemical procedures: The sections, placed on 3-aminopropyltriethoxysilane (Sigma, St.Louis, MT, USA) coated slides, were stained by streptavidin-biotin-peroxidase complex (SBPC) technique (Zymed, Histostain Plus Kit, California, USA) using polyclonal anti-enos (1/100, RB-1711, Labvision) and inos (1/100, RB-1605, Labvision). The slides were dried overnight at $37 \mathrm{oC}$; dewaxed, changing the xylene twice, with a 10 min. interval between change; rehydrated using the graded alcohol series; and placed in distilled water for $10 \mathrm{~min}$. Antigen retrieval was facilitated by heating in a citrate buffer $(\mathrm{pH}$ 6.0) for 20 min. in a microwave oven with a power of $600 \mathrm{~W}$. The slides were dipped in freshly prepared absolute methanol containing hydrogen peroxide (H2O2) $3 \% \mathrm{v} / \mathrm{v}$ for $5 \mathrm{~min}$. to block endogenous peroxidase activity. After washing with phosphate buffer solution (PBS), all sections were preincubated in a $10 \%$ normal goat serum for $30 \mathrm{~min}$. at room temperature (RT) to block nonspecific binding of the second-step antibody. Sections were incubated with primary antibody for $60 \mathrm{~min}$. at RT and were rinsed with PBS. The sections were then incubated with broad-spectrum biotin-conjugated second-step antibody (Zymed, California, USA) for $10 \mathrm{~min}$. at RT and were then rinsed in PBS. SBPC was applied for $10 \mathrm{~min}$. at room temperature. Amino ethyl carbazole (AEC) or 3,3' Diaminobenzidine (DAB) was used as chromogen in $\mathrm{H} 2 \mathrm{O} 2$ for $10 \mathrm{~min}$. (controlled by visual observation with a microscope). The sections were counterstained with Mayer's haematoxylin for $1 \mathrm{~min}$, rinsed with tap water, and mounted with an aqueous mounting medi- 
um. eNOS and iNOS activities were classified as mild $(+)$, moderate $(++)$ and severe $(+++)$.

\section{Results}

The results of the microscopic examination are summarized in (Table 1 and 2). Mild eNOS activities were seen in both diabetic and control groups at 2nd day specimens. Activities of silk and monocryl were moderate but greater than the control group at 7th day. However the activities of silk and catgut were similar both in

Table 1. Endothelial nitric oxide synthase activities of the diabetic and control groups. (-: No reaction, + : Mild, ++ : Moderate, +++ : Severe).

\begin{tabular}{|l|c|c|c|c|c|}
\hline \multicolumn{2}{|c|}{ eNOS } & $2^{\text {nd }}$ day & $7^{\text {th }}$ day & $\begin{array}{c}\mathbf{1 4}^{\text {th }} \\
\text { day }\end{array}$ & $\begin{array}{c}\mathbf{2 1}^{\text {st }} \\
\text { day }\end{array}$ \\
\hline \multirow{2}{*}{ Diabetic } & Monocryl & + & ++ & + & + \\
\cline { 2 - 6 } Group & Silk & + & ++ & + & ++ \\
\cline { 2 - 6 } & Catgut & + & ++ & ++ & +++ \\
\hline \multirow{2}{*}{ Control } & Monocryl & + & + & - & - \\
\cline { 2 - 6 } Group & Silk & + & + & + & - \\
\cline { 2 - 6 } & Catgut & + & ++ & ++ & + \\
\hline
\end{tabular}

diabetic and control groups at 14th day, this activity in monocryl was mild. 21st day specimens revealed that monocryl has mild eNOS activity than silk and catgut sutures (Fig. 1 a,b,c).

On the other hand iNOS activities of the diabetic and control groups presented differencies compared to eNOS activities of the other groups. Activities in diabetic group were greater than control groups and the degrees of the activity in monocryl, silk and catgut sutures were mild, severe and moderate at the 7th day (Fig. 2 a,b,c). At the

Table 2. Induced nitric oxide synthase activities of the diabetic and control groups. (-: No reaction, + : Mild, ++ : Moderate, +++ : Severe).

\begin{tabular}{|l|l|c|c|c|c|}
\hline \multicolumn{2}{|c|}{ iNOS } & $2^{\text {nd }}$ day & $7^{\text {th }}$ day & $\begin{array}{c}1^{\text {th }} \\
\text { day }\end{array}$ & $\begin{array}{c}\mathbf{2 1}^{\text {st }} \\
\text { day }\end{array}$ \\
\hline \multirow{2}{*}{ Diabetic } & Monocryl & + & + & - & - \\
\cline { 2 - 6 } Group & Silk & ++ & +++ & ++ & - \\
\cline { 2 - 6 } & Catgut & + & ++ & + & - \\
\hline \multirow{2}{*}{ Control } & Monocryl & + & - & - & - \\
\cline { 2 - 6 } Group & Silk & + & - & - & - \\
\cline { 2 - 6 } & Catgut & ++ & + & - & - \\
\hline
\end{tabular}
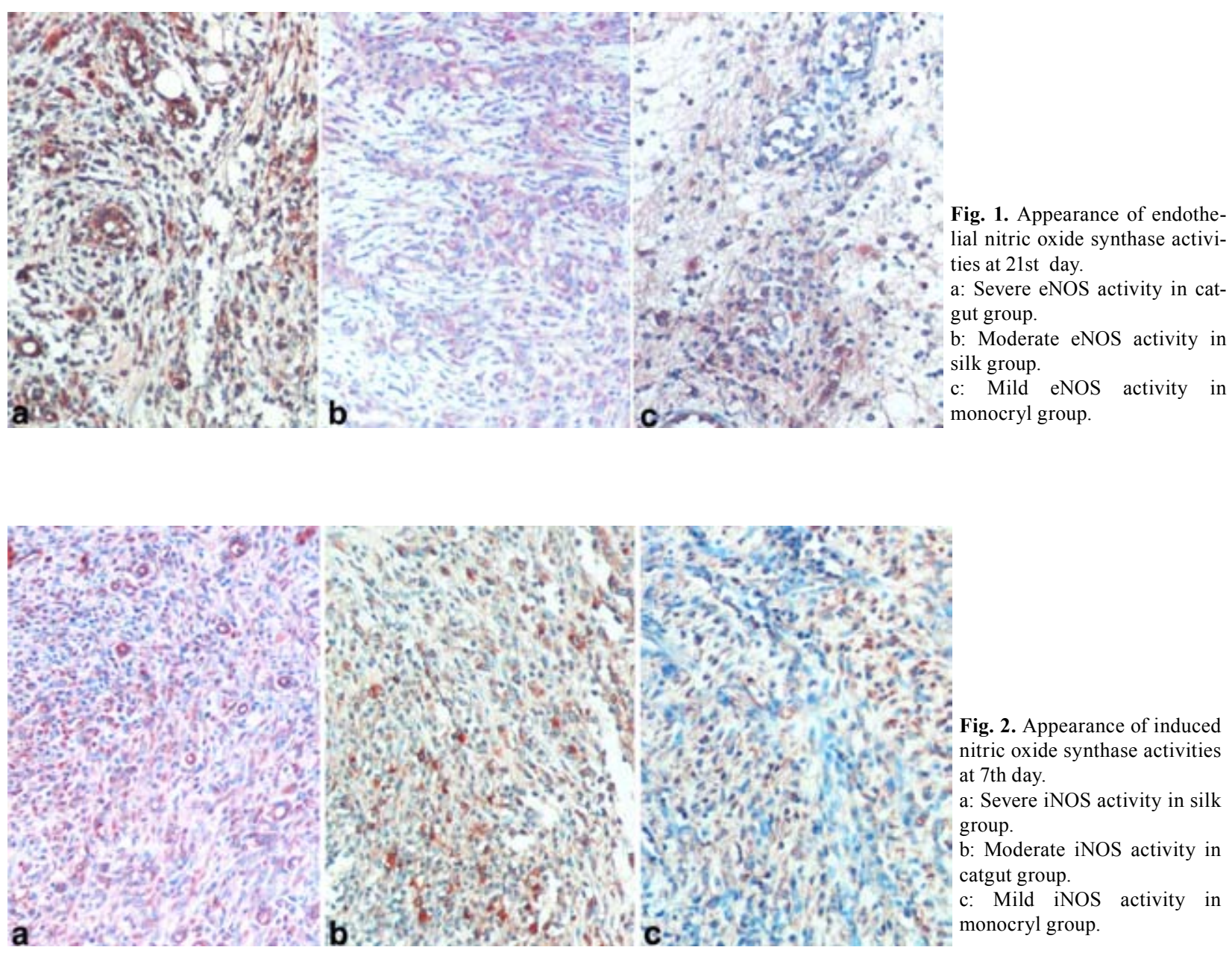
14th day there was no iNOS activity in diabetic monocryl group.

\section{Discussion}

There is much evidence to support that NO plays a major role in wound healing. Administration of NOS inhibitors results in delayed reepithelization and collagen formation(4). Several studies have implicated that NO might plays a vital role in all the phases of wound healing (8). Schaffer et al (4) suggest that nitric oxide synthesis is critical to wound collagen accumulation and acquisition of mechanical strength.

In the present study three different types of suture materials were used for comparison in the analyses of oral wound healing and NO dynamics. Monocryl showed less eNOS activity in control group at 14th day. 21st day specimens revealed that monocryl has mild eNOS activity than silk and catgut sutures in diabetic group compared to control group in the present study. iNOS activities of the diabetic and control groups presents differences compared to eNOS activities of the other groups. At the 14th day there was no iNOS activity in diabetic monocryl group.

The isoforms of NOS, eNOS and nNOS, are calcium dependent and generate low levels of NO. eNOS is expressed in endothelial cells, cardiac myocytes, and hippocampal pyramidal cells and is involved in maintaining vascular tone, inhibiting adhesion of platelets and white cells, suppressing smooth muscle cell proliferation, and promoting angiogenesis $(9,10)$. iNOS/ eNOS deficient mice show delayed healing of excisional wounds $(11,12)$. Moreover impaired wound healing in diabetics has been associated with reduced NO synthesis. Thus high level of bioavailable NO in wounds is important for enhancing the healing process (13). The inducible isoform (iNOS) is synthesized in the early phase of wound healing by inflammatory cells, mainly macrophages. However many cells participate in NO synthesis during the proliferative phase after wounding $(4,5)$. Neutrophils and macrophages play a crucial role in the inflammatory and proliferative stages of wound healing(1).

Loss of a functional healing process could lead to severe disabilities (3). It is known that diabetes is a systemic disorder that affects almost all body systems, either directly or indirectly through its complications. Although diabetes is a chronic progressive disease, acute diabetes can be associated to subclinical alterations, and responsible for deficiencies in defense cells and in repair tissue failures. Among the acute complications, acute metabolic derangements, urinary tract infections, skin and other infections and side effects of drugs are important. However the majority of cells are dependent on insulin for the intracellular shift of glucose, which is the major source of energy for cellular functions (14).
Although the diabetes is a chronic systemic disorder, the relationship of different suture materials and nitric oxide dynamics was investigated on short-term induced diabetic rats model in the present study. Romana-Souza et al. (15) reported that the model of acute streptozotocin-induced diabetes which does not have the metabolic or pathologic features of long-standing human diabetes, but provides a good and analogous model to study acute wound healing events. Also we used the same diabetic rat model as mentioned by Romana-Souza et al. (15). In the present study, the diabetic rats developed typical physiological characteristics observed in diabetic humans such as hyperglycemia, weight loss and poor wound healing.

The wound healing process is involves a complex, dynamic series of events including clotting, inflammation, granulation tissue formation, epithelialization, neovascularization, collagen synthesis, and wound contraction (1).

It is important that local inflammatory response reduce in diabetic patients. The effects of iNOS in the inflammatory response are particularly complex, and the enzyme appears to be involved in both promotion and resolution of inflammation (10). Diabetic rats have decreased NO2- +NO3- in wound fluid as well as impaired breaking strength and decreased collagen content of healing wounds. Interestingly, insulin treatment not only improves wound tensile strength and collagen deposition in these animals, but also partially restores NO production (16). Contrary to what is seen in rodent models, increased levels of iNOS and eNOS protein and enzymatic activity have been reported in human diabetic foot ulcers (compared to normal skin from normal or diabetic patients), and these patients have increased plasma NO2- +NO3- (13). In contrast to the findings of Jude et al. (13), Schwenter et al. (8) suggested that iNOS mRNA may in fact be underexpressed in diabetic patient foot ulcers. In contrast to rat studies in the present study; iNOS and eNOS activities were increased immunohistochemically.

Sutures used in oral surgery behave differently from other parts of the body due to the quality of the tissues involved, presence of saliva and specific microbiota. They represent a pathway communicating the internal and external regions of the tissues, influencing the quality of wound healing (2). It has been mentioned that rather than factors related to suture materials and different surgical techniques, and with the exception of surgeon experience, general characteristics of the patients (i.e., sex and age) and of the wounds (i.e., length and site) seemed to be primarily responsible for local wound complications (17).

Biological behavior must be considered during the selection of the suture material to be used in oral surgery. Several studies have demonstrated and confirmed a 
reduced inflammatory response after the application of monofilament suture materials in oral wounds compared to multifilament ones $(18,19)$. In accordance with the literature $(19,20)$ in the present study, monocryl which is a monofilament suture, created less reaction compared with catgut and silk which are in polyfilament character.

As a result, in diabetic groups, monocryl presented a lower NOS activity than the other materials immunohistochemically. All of the materials have the potential capacity to be well tolerated however monocryl seemed to have more benefical effects on wound healing in diabetic subjects.

\section{References}

1. Singer AJ, Clark RA. Cutaneous wound healing. N Engl J Med. 1999;341:738-46.

2. Certosimo FJ, Nicoll BK, Nelson RR, Wolfgang M. Wound healing and repair: a review of the art and science. Gen Dent. 1998;46:362-9.

3. Bello YM, Phillips TJ. Recent advances in wound healing. JAMA. 2000;283:716-8.

4. Schaffer MR, Tantry U, Gross SS, Wasserburg HL, Barbul A. Nitric oxide regulates wound healing. J Surg Res. 1996;63:237-40.

5. Luo JD, Chen AF. Nitric oxide: a newly discovered function on wound healing. Acta Pharmacol Sin. 2005;26:259-64.

6. Carew JF, Ward RF, LaBruna A, Torzilli PA, Schley WS. Effects of scalpel, electrocautery, and $\mathrm{CO} 2$ and KTP lasers on wound healing in rat tongues. Laryngoscope. 1998;108:373-80.

7. Sinha UK, Gallagher LA. Effects of steel scalpel, ultrasonic scalpel, $\mathrm{CO} 2$ laser, and monopolar and bipolar electrosurgery on wound healing in guinea pig oral mucosa. Laryngoscope. 2003;113:228-36. 8. Schwentker A, Vodovotz Y, Weller R, Billiar TR. Nitric oxide and wound repair: role of cytokines? Nitric Oxide. 2002;7:1-10.

9. Alcaraz MJ, Guilln MI. The nitric oxide related therapeutic phenomenon: a challenging task. Curr Pharm Des. 2002;8:215-31.

10. Vallance P, Leiper J. Blocking NO synthesis: how, where and why? Nat Rev Drug Discov. 2002;1:939-50.

11. Lee PC, Salyapongse AN, Bragdon GA, Shears LL 2nd, Watkins $\mathrm{SC}$, Edington HD, et al. Impaired wound healing and angiogenesis in eNOS-deficient mice. Am J Physiol. 1999;277:H1600-8.

12. Yamasaki K, Edington HD, McClosky C, Tzeng E, Lizonova A, Kovesdi I, et al. Reversal of impaired wound repair in iNOS-deficient mice by topical adenoviral-mediated iNOS gene transfer. J Clin Invest. 1998;101:967-71.

13. Jude EB, Boulton AJ, Ferguson MW, Appleton I. The role of nitric oxide synthase isoforms and arginase in the pathogenesis of diabetic foot ulcers: possible modulatory effects by transforming growth factor beta 1. Diabetologia. 1999;42:748-57.

14. McMurry JF Jr. Wound healing with diabetes mellitus. Better glucose control for better wound healing in diabetes. Surg Clin North Am. 1984;64:769-78.

15. Romana-Souza B, Nascimento AP, Monte-Alto-Costa A. Propranolol improves cutaneous wound healing in streptozotocin-induced diabetic rats. Eur J Pharmacol. 2009;611:77-84.

16. Schäffer MR, Tantry U, Efron PA, Ahrendt GM, Thornton FJ, Barbul A. Diabetes-impaired healing and reduced wound nitric oxide synthesis: a possible pathophysiologic correlation. Surgery. 1997; 121:513-9.

17. Gabrielli F, Potenza C, Puddu P, Sera F, Masini C, Abeni D. Suture materials and other factors associated with tissue reactivity, infection, and wound dehiscence among plastic surgery outpatients. Plast Reconstr Surg. 2001;107:38-45.

18. Lilly GE, Armstrong JH, Salem JE, Cutcher JL. Reaction of oral tissues to suture materials. II. Oral Surg Oral Med Oral Pathol.
1968;26:592-9.

19. Postlethwait RW. Long-term comparative study of nonabsorbable sutures. Ann Surg. 1970;171:892-8.

20. Nary Filho H, Matsumoto MA, Batista AC, Lopes LC, de Góes FC, Consolaro A. Comparative study of tissue response to polyglecaprone 25, polyglactin 910 and polytetrafluorethylene suture materials in rats. Braz Dent J. 2002;13:86-91. 